\title{
Monitoring player fitness, fatigue status and running performance during an in-season training camp in elite Gaelic football
}

\author{
Shane Malone \\ Technological University Dublin, Shane.Malone@TUDublin.ie \\ Brian Hughes \\ Technological University Dublin \\ Mark Roe \\ Technological University Dublin
}

See next page for additional authors

Follow this and additional works at: https://arrow.tudublin.ie/ittsciart

Part of the Sports Sciences Commons

\section{Recommended Citation}

Shane Malone, Brian Hughes, Mark Roe, Kieran Collins \& Martin Buchheit (2017) Monitoring player fitness, fatigue status and running performance during an in-season training camp in elite Gaelic football, Science and Medicine in Football, 1:3, 229-236, DOI: 10.1080/24733938.2017.1361040

This Article is brought to you for free and open access by the School of Science and Computing at ARROW@TU Dublin. It has been accepted for inclusion in Articles by an authorized administrator of ARROW@TU Dublin. For more information, please contact arrow.admin@tudublin.ie, aisling.coyne@tudublin.ie, gerard.connolly@tudublin.ie.

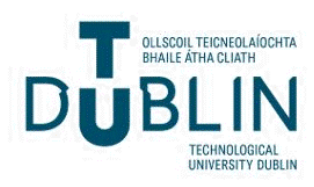


Authors

Shane Malone, Brian Hughes, Mark Roe, Kieran Collins, and Martin Buchheit

This article is available at ARROW@TU Dublin: https://arrow.tudublin.ie/ittsciart/121 
Monitoring player fitness, fatigue status and running performance during an in-season training camp in elite Gaelic football

\author{
Shane Malone ${ }^{1,2}$ Brian Hughes $^{2}$, Mark Roe ${ }^{2,3}$, Kieran Collins ${ }^{2}$, Martin \\ Buchheit $^{4}$
}

1. The Tom Reilly Building, Research Institute for Sport and Exercise Sciences, Liverpool John Moores University, Henry Cotton Campus, 15-21 Webster Street, Liverpool, L3 2ET 2. Gaelic Sports Research Centre, Department of Science, Institute of Technology Tallaght, Tallaght, Dublin, Ireland. ${ }^{3 .}$ School of Public Health, Physiotherapy and Sports Science, Health Sciences Centre, University College Dublin, Belfield, Dublin 4. 4. Performance Department, Paris Saint Germain FC, 4A Avenue Kennedy, Saint-Germain-en-Laye, Paris, France

Running Title: Monitoring training load during in a training camp

Corresponding author: Shane Malone

c/o The Tom Reilly Building, Research Institute for Sport and Exercise Sciences, Liverpool John Moores University, Henry Cotton Campus, 15-21

Webster Street,

Liverpool, L3 2ET

Email: shane.malone@mymail.ittdublin.ie Tel: (+353) 87-4132808

Abstract word count: 250

Word count: 3510

Number of tables and figures: 4 Figures; 1 Table 


\section{ABSTRACT}

The current investigation examined selected perceptual and physiological measures to monitor fitness, fatigue and running performance during a one week in-season training camp in elite Gaelic football. Twenty-two elite Gaelic football players were monitored for training load (session RPE x duration), perceived ratings of wellness (fatigue, sleep quality, soreness); heart rate variability (HRV;LnSD1), heart rate recovery (HRR), exercise heart rate (HRex), lower limb muscular power (CMJ) and global positioning system (GPS) variables. The Yo-Yo intermittent recovery test level 1 (YoYoIR1) was assessed pre-and post the training camp. GPS units were used to monitor players throughout the camp period, with specific small sided games (SSG) used as a measure of running performance. There were significant day-to-day variations in training load measures (Coefficent of variation, $\mathrm{CV}: 51 \% ; \mathrm{p} \leq 0.001)$, HRex decreased (-12.2\%), HRR increased $(+10.3 \%)$ CMJ decreased $(-8.1 \%)$ and pre-training LnSD1 (+14.1\%) increased during the camp period. Yo-YoIR1 performance $(+19.7 \%)$, total distance (TD) $(+9.4 \%)$, high speed distance (HSD) $(+12.1 \%)$ and sprint distance (SPD) (+5.8\%) within SSG improved as the camp progressed. $\Delta$ HRex and $\Delta$ HRR were correlated with $\Delta$ Yo-YoIR $1(\mathrm{r}=0.64 ;-0.55), \Delta H S D$ $(\mathrm{r}=0.44 ;-0.58), \Delta \mathrm{SPD}(\mathrm{r}=0.58 ;-0.52) . \Delta \mathrm{LnSD} 1$ was correlated with $\Delta$ Yo-YoIR1 $(\mathrm{r}=0.48 ; 90 \% \mathrm{CI}: 0.33$ to 0.59$)$ and $\Delta \mathrm{TD}(\mathrm{r}=0.71)$ There were large correlations between $\Delta$ wellness and $\Delta$ Yo-YoIR1 $(r=0.71), \Delta \mathrm{TD}(\mathrm{r}=$ $0.68)$ and $\triangle \mathrm{SPD}(\mathrm{r}=0.68)$. Increases in training load were observed during the training camp. Daily variations in training load measures across the camp period were shown to systematically impact player's physiological, performance and wellness measures.

Keywords: GPS, HR, Team-sports, Monitoring, Training Load 


\section{INTRODUCTION}

Gaelic football is an intermittent team based field sport that can be best described as a running game that requires a combination of athleticism with skilful foot and hand passing. Players complete on average $9222 \pm$ 1588 -m of total running distance with $18 \%$ completed at high speed $(\geq 17$ $\mathrm{km} \cdot \mathrm{h}^{-1}$ ) across $70 \mathrm{~min}$ of match-play (Malone et al., 2016c; Malone, Solan $\&$ Collins 2016a). The monitoring of training load within all team sports is important for the periodisation and subsequent planning of the physical 'dose' during training periods (Malone \& Collins, 2016; Tran et al., 2015). This is of further importance within condensed acute training periods such as in-season training camps. Within team sports (Gabbett et al., 2012; Rogalski et al., 2013; Ritchie et al., 2016) reductions in training load as the season progresses is commonplace. However, within Gaelic football previous literature has shown no changes in training load across the season (Malone et al., 2016b). The seasonal calendar is heavily focused towards the end of year All-Ireland series (Malone et al., 2016b). The All-Ireland series represents a direct knock-out style competition that takes place after the National League competition during the months of May through to September, and is considered the sports premier competition. The All-Ireland series is the key factor in the lack of variation seen in training load across the calendar within Gaelic football (Malone et al., 2016b). In order to maximise adaptations prior to the beginning of this competition teams regularly participate in an acute intensified training period during a training camp. Anecdotally, teams treat these camps as professional environments training two or three times daily with as much as 10 sessions completed during a weekly period.

Many monitoring variables have been suggested to analyse players training load and status (Buchheit et al., 2012; Buchheit, 2014; Thorpe et al., 2015). However, their invasive and/or exhaustive nature makes their frequent assessment within team sports difficult. Non-invasive measures of assessing fitness, wellness, recovery status and physical performance have received increased interest over the last number of years (Le Meur et al., 2013; Le Meur et al., 2016). These measures of interest include, sub-maximal exercise 
HR (HRex) and pre-exercise cardiac autonomic activity as inferred from heart rate variability (HRV) measures, simply defined as the variation in the beat-to-beat intervals of the heart (Le Meur et al., 2013; Le Meur et al., 2016). When considering non-invasive performance assessment $\mathrm{HR}_{\mathrm{ex}}$, considered an index of cardiorespiratory fitness has previously been strongly correlated with running performance (Buchheit et al., 2010). HRV has previously been shown to be related to acute fatigue experienced by players following bouts of exercise (Le Meur et al., 2013; Le Meur et al., 2016) while also allowing coaches to alter the training periodization of athletes (Le Meur et al., 2013; Le Meur et al., 2016). Heart rate recovery (HRR) can infer how athletes are adapting to a specific training stimulus (Buchheit, 2014) and has been reported to be sensitive to functional overreaching (Le Meur et al., 2016). Finally, psychological monitoring is also purported to be an effective means of assessing players' responses to subtle variations in training load (Gallo et al., 2016; Thorpe et al., 2015; Main \& Grove, 2009). However, whether these variables are sensitive to acute fatigue, wellness, recovery, status and in turn, fitness, during an in-season training camp within elite Gaelic football players is unknown.

Despite the lack of Gaelic football specific research, across team sports numerous descriptive analyses of training camps have been conducted (Buchheit et al., 2013; Pitchford et al., 2016; Thornton et al., 2016). Recently research revealed that during a camp period training loads can increase by between 50-58\% when compared to normative training load values (Buchheit et al., 2013; Thornton et al., 2016). Not surprisingly, during these acute intensified periods, players have been found to have disturbed sleep patterns and reduced wellness measures (Thornton et al., 2016). Indeed, these intensified periods result in training-induced fatigue which is generally associated with an increased sympathetic activity (Mourot et al., 2004) that can increase sub-maximal HR and decrease HRV measures (LnSD1) within players (Buchheit, 2014). Interestingly despite these acute increases in training load causing the early stages of over-reaching in athletes, these camp 
periods tend to increase players performance measures (Buchheit et al., 2013).

Given the lack of research conducted on camp periods in elite Gaelic football, the overall purpose of the current study was to (1) examine the daily variations of selected running, physiological and psychometric variables during an in-season training camp in elite Gaelic football players to (2) examine the usefulness of these variables in monitoring players training responses during an intensified training period and (3) to assess these variables association with changes in Yo-YoIR1 and standardised small sided games performance during the camp period.

\section{METHODS}

\section{Subjects}

The current investigation was a observational study of elite Gaelic football players competing at the highest level of competition (National League Division 1 and All-Ireland). Data were collected for 22 players (Mean $\pm S D$, age: $24.3 \pm 6.1$ years; height: $180.2 \pm 7.3 \mathrm{~cm}$; mass: $81.6 \pm 7.5$ $\mathrm{kg}$ ) across a one-week training camp during the competition season. The senior level playing experience of the current squad was $8.5 \pm 4.3$ years. The study was approved by the local institute's research ethics committee and written informed consent was obtained from each participant.

\section{Training Camp}

The study was conducted during a one-week training camp (7-day) prior to the commencement of the All-Ireland series. During the one-week training camp, all players took part in an intensified team based training period as prescribed by the coaches and strength and conditioning staff. Players participated in 10 field based sessions (6 technical, 2 fitness and skill based sessions, 2 match play sessions, total session exposure: $11.5 \mathrm{hr}$ ), 2 interval cycling sessions (10-15 maximal efforts repetitions of 5-30 seconds in duration x 3-6 sets) (total session exposure: $1.5 \mathrm{hr}$ ) and two strength based 
gym sessions (total session exposure: $2.5 \mathrm{~h}$ ). All players were provided with standardised post training session nutritional plan by the team's nutritionist. All plans were developed and tailored to each individual athlete's needs to ensure adequate fluid and nutrient intake and recovery between sessions.

\section{Monitoring Load and Wellness}

The intensity of all training sessions were estimated using the modified Borg CR-10 rate of perceived exertion (RPE) scale, with ratings obtained from each individual player $30 \mathrm{~min}$ after the end of each training session (Malone et al., 2016b; Fanchini et al., 2016). Each individual RPE value was multiplied by the session duration to generate an arbitrary unit (AU) internal training load score for the specific session (Malone et al., 2016c). Additionally, a psychometric questionnaire was used to assess general indicators of player wellness (Gallo et al., 2016; Thorpe et al., 2015; Main \& Grove, 2009). The questionnaire assessed the following elements of wellness: 1) muscular soreness, 2) sleep quality, 3) fatigue, 4) stress and 5) energy level, on a seven-point likert scale ranging from 1 (strongly disagree) to 7 (strongly agree). The five individual wellbeing responses for a given day were summed to provide a quantitative score of overall perceived wellness for each player with a maximal wellbeing score of 35 arbitrary units (AU). The co-efficient of variation for the five indices ranged from 5-11\% within the current squad. Prior to training players completed an assessment for vertical jump performance through a countermovement jump (CMJ) assessment (OptoJump, Microgate, Bolzano, Italy), in which they were required to perform a single $\mathrm{CMJ}$. The $\mathrm{CMJ}$ were performed with hands held firmly on the hips and subjects were instructed to jump as high as possible. The jump was performed at a self-selected countermovement depth and no instruction was given on what countermovement depth to use with flight time used to estimate jump height $(\mathrm{cm})$.

\section{Monitoring Fitness}

A Yo-Yo Intermittent Recovery test level 1 (Yo-YoIR1) (Bangsbo et al., 2008; Roe and Malone, 2016) was performed outdoors at the same time 
of day (11:00) in temperate conditions $\left(20^{\circ} \mathrm{C}\right)$, at the beginning (day 1$)$ and at the end (day 7) of the camp. All players were familiar with this test, as it was part of the regular fitness testing battery implemented by the conditioning staff. Briefly, the Yo-YoIR1 consists of repeated 20-m shuttle runs at increasing speeds (starting at $8 \mathrm{~km} \cdot \mathrm{h}^{-1}$ ) with $10 \mathrm{~s}$ of active recovery (consisting of 5-m of jogging) between runs, until exhaustion. A submaximal 5-min running/5-min recovery test (Buchheit et al., 2013; Buchheit et al., 2010) was performed at the start of every training/testing session to assess training status. All players were tested simultaneously with the intensity of the exercise bout fixed at $13 \mathrm{~km} \cdot \mathrm{h}^{-1}$ over $40-\mathrm{m}$ shuttles. HRex and post exercise heart rate recovery (HRR) for a $1 \mathrm{~min}$ period were recorded during the assessment (Le Meur et al., 2016). Prior to all training HRV (standard deviation of instantaneous beat-to-beat $\mathrm{R}-\mathrm{R}$ interval variability) was measured by all players using a provided HR strap (Polar Team 2 system; 1.4.1, Polar Electro Oy, Kempele, Finland). Specifically, in a room by themselves prior to training, players were instructed to remain in a supine position for $8 \mathrm{~min}$. R-R intervals were recorded and analysed during the last 4 min of the supine position (Le Meur et al., 2013).

During all outdoor training sessions' players were monitored using GPS units (18-Hz, GPEXE LT, Exelio, Udine, Italy). Sport-specific running performance was assessed using specific small-sided games (SSG) that were completed every day during the camp as the first main drill of training. The specific SSG was a 4v4 - 60x20-m - touchdown drill (Malone, Solan \& Collins, 2016a), where the aim of the drill was for teams to keep possession of the ball and attack an end zone area. Once a team had moved the ball into the end zone area they retain possession of the ball and aimed to move the ball back down into the opposite end zone. Total (TD); high-speed (HSD, $\geq 17 \mathrm{~km} \cdot \mathrm{h}^{-1}$ ), sprint (SPD; $\geq 22 \mathrm{~km} \cdot \mathrm{h}^{-1}$ ) distance as well as maximal velocity $\left(\mathrm{km} . \mathrm{h}^{-1}\right)$ were evaluated during all training sessions. Training data was analysed post session with retrospective analysis conducted on all sessions. Each file was then trimmed so only data recorded during each session and 
specifically each drill when the player was on the field was included for further analysis. Data were exported into a customised spreadsheet (Excel, Microsoft Redmond, USA). This spreadsheet allowed for the analysis of distance covered in the following categories: total distance (TD; $\mathrm{m}$ ); high speed running $\left(\geq 17 \mathrm{~km} \cdot \mathrm{h}^{-1}, \mathrm{HSD} ; \mathrm{m}\right)$, sprint distance $\left(\geq 22 \mathrm{~km} \cdot \mathrm{h}^{-1} ; \mathrm{SPD} ; \mathrm{m}\right)$, and maximal velocity $\left(\mathrm{km} \cdot \mathrm{h}^{-1}\right)$.

Statistical Analysis

Data are presented as means $( \pm \mathrm{SD})$ and correlations as means $(90 \%$ 

\pm 4254 -m with $9244 \pm 2254-\mathrm{m}$ of HSD and $1678 \pm 554$-m of SPD. Players completed twelve training sessions across a seven-day camp period with a training load (AU) of $5984 \pm 554$ AU. Changes in training load and status measures are shown in Figure 1 with changes in wellness and status measures 
The aim of the current investigation was to examine selected movement, physiological and perceptual measures to monitor fitness, fatigue and running performance during an in-season training camp in elite Gaelic football players. The main findings of the current study were (1) that running performance during SSG and Yo-YoIR1 performance increased throughout the camp period. (2) Heart rate (HRR, HRex, LnSD1), all wellness and vertical jump performance $(\mathrm{CMJ})$ measures were shown to respond to subtle daily changes in training load during the period. (3) Changes in heart rate measures were correlated to changes in player wellness during the camp. (4) Changes in wellness and heart rate measures were correlated to changes in Yo-YoIR1 performance in addition to running performance during standardised SSG during the camp.

Our results show that during the one-week training camp players completed on average loads of $5984 \pm 554$ AU across twelve training sessions (Figure 1). The current workloads are higher than previously reported within Gaelic football (2560-2740 AU) (Malone et al., 2016b). Interestingly, within the current weekly period loads were $42-45 \%$ higher on average, however, this is not surprising and agrees with research conducted during many other training camp periods in team sports (Buchheit et al., 2013; Thornton et al., 2016). Coaches should be aware that sudden increases or "spikes" in load have been linked to increased risk of injury within Gaelic football (Malone et al., 2016b; Malone et al., 2016c) and other team sport cohorts (Cross et al., 2016; Malone et al., 2016a). Therefore, coaches need to plan for these in-season camp periods ensuring that players' previous loading prior to the camp period is appropriate in order to best moderate the risk associated with the increased training demand placed on players during these training camp periods (Malone et al., 2016b; Malone et al., 2016c). Furthermore, and in contrast to previous literature where spikes in load have been linked to injury risk (Malone et al., 2016a; Cross et al., 2016; Malone et al., 2016b), in the current study, no injuries were suffered by players monitored for the whole duration of the camp, this may be related to the reduction in overall maximal velocity and lower limb power capabilities of players which may have reduced the overall intensity of training sessions. 
During the camp, player's wellness measures did not significantly decrease from the start to the end. Moreover, when LnSD1 and HRR were considered as a cardiac autonomic marker of physiological stress throughout the period, we observed an increase across the aforementioned period (Figure 1). Previous studies have suggested that LnSD1 and HRR should decrease in the presence of fatigue and physiological stress. However, recently several studies have shown that in the presence of systematic increases in training load that a down-regulation of the sympathetic nervous system and/or changes in the balance between parasympathetic and sympathetic tone can occur. The down-regulation can result in increased pre-exercise LnSD1 and post-exercise HRR responses, this has been partly linked to an increase in parasympathetic modulation of HR during the overload period (Le Meur et al., 2016; Buchheit, 2014; Le Meur et al., 2013). Therefore, practitioners should be aware that the utilisation of a single measure of physiological training would not be recommended to monitor responses to intensified training load periods in team sports such as Gaelic football. We therefore suggest a holistic approach to monitoring responses to intensified periods of training where a number of measures are utilised by practitioners in order to modify and adjust players training load to ensure players are in a nonfatigued state prior to competition.

During the current camp period TD, HSD and SPD improved during a standardized SSG with improved Yo-YoIR1 performance during the camp period (Figure 3). While a lack of a control group prevents definitive conclusions to be made about the acute intensified training period, these results may provide efficacy for such a camp to improve running and physical performance characteristics of elite Gaelic football players. These results have direct implications for Gaelic football coaches who are searching for the most prudent training strategies to apply during in-season training camps. The improvement in running performance during performance tests such as Yo-YoIR1 can be related to general traininginduced improvements in fitness and wellness. Furthermore, the improvements in SSG running performance may be related to the increase in aerobic fitness during the training camp. However, familiarisation with the 
drill type as the camp progressed may be considered as a potential impacting factor within the current results, however future literature needs to investigate this in greater detail to confirm the authors hypothesis (Figure 3). Moreover, moderate to very-large correlations between heart rate training load variables (HRex, HRR, LnSD1) and changes in SSG running and YoYoIR1 performance were observed. These findings are in agreement with previous training camp investigations (Buchheit et al., 2013). These findings add support to the utilisation of simple, non-invasive and non-fatiguing measures for monitoring training responses in elite team sport athletes. Interestingly, the current study found a linear increase in standardised SSG running performance across the duration of the training camp. Although the magnitude of increase for these running based variables was lower than that observed in Yo-YoIR1 performance. Additionally, the correlations between changes in running and HR measures was lower than that of Yo-YoIR1. We suggest that standardised SSG may provide an insight into potential acute changes in physical performance of team sport players. We suggest that future studies in Gaelic football assess the application of standardised SSG as a potential running performance test during training periods. However, we acknowledge that a stringent prediction of changes to physical performance characteristics during standardised SSG from physiological and running measures is more difficult than in standardised testing protocols such as the Yo-YoIR1.

Significant daily variations in training load (sRPE) across the camp period were observed and these measures were shown to systematically impact player's physiological response, psychological wellness and running performance for the following day. The current findings have significant implications for Gaelic football coaches highlighting the importance of systematic monitoring of players. Additionally, both negative and positive correlations between these daily fluctuations in training load variables and changes in load measures were found. The negative association between changes in training load and wellness measures was expected. Interestingly, we observed that increases in training load were positively related to changes in HRR and LnSD1 (i.e. increases in acute load resulted in increased HR 
responses). These results may be related to acute training load fatigue which may have resulted in a modulation of HR responses and reduced player's sympathetic activity (Le Meur et al., 2013; Le Meur et al., 2016).

The findings within the current investigation provide evidence supporting the sensitivity of simplistic monitoring measures to detect acute fluctuations in training load. Moreover, the findings show that the collection of training load data within Gaelic football players even when considered across an acute period (7 days) can provide meaningful indirect information about player's responses and status to fluctuations in training load the following day. Interestingly we found significant reductions in self-reported sleep quality of players throughout the camp period (Figure 2). The finding agrees with previous investigations in rugby league (Thornton et al., 2016) and Australian Rules cohorts (Buchheit et al., 2013; Pitchford et al., 2016), that reported reductions in sleep efficiency and the onset of sleep during training camp periods when contrast to home based training periods. The addition of our findings to this previously published research demonstrates that sleep quality is jeopardized during training camps (Pitchford et al., 2016). We suggest that Gaelic football coaches' prioritise periods of planned sleep such as naps for players during these camp periods to reduce the effects of acute fatigue. Previous studies (Thornton et al., 2016) reported that when athletes adhered to napping recommendations during a training camp, there were benefits in recovery and subsequent night time sleep quality. Optimal quality and quantity of sleep would seem beneficial given its established role in facilitating athletic recovery and performance (Pitchford et al., 2016; Thornton et al., 2016). Indeed, improving sleep quality within basketball players improved sprint time, free throw accuracy, reaction time and ratings of physical and wellness (Mah et al., 2011). Theoretically, maximising sleep time and quality during a period of high training stress may accentuate training recovery and adaptations (Pitchford et al., 2016; Thornton et al., 2016).

The findings of the current paper need to be considered with several limitations. Firstly, since training sessions with different contents were 
carried out during the training camp, it is difficult to determine whether the whole training camp or only some of the training sessions were responsible for the induced changes in the measured variables. Furthermore, the investigation failed to provide a control group to compare a similar period of intensified training to in order to determine if the findings are consistent across similar cohorts and time frames. Future research should examine in

\section{CONCLUSION} detail the changes in both training load characteristics and wellness profiles of Gaelic footballers from home to camp periods. This will allow coaches to best prepare players for the increased training loads experienced during camp periods. Additionally, although no injuries were suffered by players during the current camp, future research should aim to provide an analysis of a post camp periods and account for any injuries or illnesses suffered by players following the training camp. This will facilitate optimal planning post the intensified period of training. Finally, with the observed reduction in sleep quality throughout the 7-day camp period it is important for research to examine the individual sleep responses to training load during a season but also within acute intensified periods of training.

The current camp resulted in a $42-45 \%$ increase in training load. Daily variations in training load measures across the camp period were shown to have a fluctuating impact player's physiological, performance and wellness measures from day-to-day. During the current camp period running performance measures were shown to improve during a standardized SSG with improved Yo-YoIR1 performance during the camp period. When considering the association between changes in running performance and changes in training load variables moderate to very-large correlations between heart rate variables, wellness and changes in sport specific running and YoYoIR1 performance were shown. Overall the study highlights the need to systematically monitor players while adding further credence to the application of simple, non-invasive and non-fatiguing measures for monitoring training responses in elite team sport athletes. 
- During the training camp periods heart rate (HRR, HRex, LnSD1), all wellness and vertical jump performance (CMJ) measures may be used by practitioners during training camps to identify positive and negative responses during training camp periods.

- Changes in heart rate measures were correlated to changes in player wellness, while both measures were related to changes in running performance and aerobic fitness. It may be suggested that one of these monitoring variables be used during a training camp period given the high levels of correlation.

- Monitoring high speed and sprint running distance on a day-by-day basis (e.g. GPS measures) is valuable to confirm the potential transfers from sportspecific training (SSG) to physical running performance (Yo-YoIR1) within camp settings.

\section{AKNOWLEDGEMENTS}

We wish to thank the management, coaching staff and players for their assistance and willingness to participate in the current investigation. There was no funding provided for this project, and there are no conflicts of interest.

DISCLOSURE STATEMENT

No potential conflict of interest was reported by the authors.

\section{FUNDING}

No grant support was provided for this study. 


\section{REFERENCES}

Bangsbo J, Iaia FM, Krustrup P. The Yo-Yo intermittent recovery test: a useful tool for evaluation of physical performance in intermittent sports. Sports Med 2008; 38(1):37-51.

Buchheit M. Monitoring training status with HR measures: do all roads lead to Rome? Front Physiol. 2014;5:73

Buchheit M, Mendez-Villanueva A, Quod MJ, Poulos N, Bourdon P. Determinants of the variability of heart rate measures during a competitive period in young soccer players. Eur J Appl Physiol 2010; 109:869-878.

Buchheit M, Racinais S, Bilsborough J, Bourdon PC, Voss SC, Hocking J, Cordy J, Mendez-Villanueva A, Coutts AJ. Monitoring fitness, fatigue and running performance during a pre-season training camp in elite football players. J Sci Med Sport. 2013;16(6):550-555.

Buchheit M, Simpson MB, Al Haddad H, Bourdon PC, Mendez-Villanueva A. Monitoring changes in physical performance with heart rate measures in young soccer players. Eur $J$ Appl Physiol 2012; 112(2):711-723.

Cross MJ, Williams S, Trewartha G, Kemp SP, Stokes KA. The influence of in-season training loads on injury risk in professional rugby union. Int J Sports Physiol Perform,2016; 11(3):350-355, doi: 10.1123/ijspp.2015-0187

Franchini M, Ferraresi I, Petruolo A, Azzalin A, Ghielmetti G, Schena F, Impellizzeri FM. Is a retrospective RPE appropriate in soccer? Response shift and recall bias. Sci Med football 2016; Aug. doi: 10.1080/02640414.2016.1231411

Gabbett TJ, Jenkins DG. Relationship between training load and injury in professional rugby league players. J Sci Med Sport. 2011;14(3):204-209.

Gallo, TF, Cormack SJ, Gabbett TJ, Lorenzen CH. Pre-training perceived wellness impacts training output in Australian football players. J Sports Sci 2016; 34(15): 1445-1451.

Hopkins WG, Marshall SW, Batterham AM, Hanin J. Progressive statistics for studies in sports medicine and exercise science. Med Sci Sports Exerc 2009; 41(1):3-13.

Huikuri HV, Seppanen T, Koistinen MJ, Airaksinen J, Ikaheimo MJ, Castellano A, Myerburg RJ. Abnormalities in beat-to-beat dynamics of heart rate before the spontaneous onset of life-threatening ventricular tachyarrhythmias in patients with prior myocardial infarction. Circulation 1996; 93(10):1836-1844.

Le Meur Y, Pichon A, Schaal K, Louis J, Gueneron J, Vidal PP, Hausswirth C. Evidence of parasympathetic hyperactivity in functionally overreached athletes. Med Sci Sports Exerc. 2013;45:2061-71.

Le Meur Y, Buchheit M, Aubrey A, Coutts AJ, Hausswirth C. Assessing overreaching with HRR: What is the minimal exercise intensity required? Int J Sports Physiol Perform. 2016; In Press. 
Mah CD, Mah KE, Kezirian EJ, Dement WC. The effects of sleep extension on the athletic performance of collegiate basketball players. Sleep 2011;34(7):943-950.

Main LC, Grove JR. A multi-component assessment model for monitoring training distress among athletes. Euro J Sport Sci. 2009; 9(4), 195-202. doi:10.1080/1746139090 2818260

Malone S, Collins K. Relationship between individualised training impulse and aerobic fitness measures in hurling players across a training period. J Strength Cond Res, 2016. doi: 10.1519/JSC.0000000000001386. Epub ahead of print

Malone S, Owen A, Newton M, Mendes B, Collins KD, Gabbett TJ. The acute:chronic workload ratio in relation to injury risk in professional soccer. J Sci Med Sports 2016a; pii:s1440-2440(16)30230-30234. doi:10.1016/j.jsams.2016.10.014. In Press.

Malone S, Solan B, Collins K, Doran D.A. The positional match running performance of elite Gaelic football. $J$ Strength Cond Res. 2016a; 30(8): 2292-2298. doi: 10.1519/JSC.0000000000001309.

Malone S, Solan B, Collins D. The influence of pitch size on running performance during Gaelic football small sided games. Int J Perform Anal Sport 2016b; 16 (1): 111-121.

Malone S, Solan B, Collins K. The running performance profile of elite Gaelic football matchplay. J Strength Cond Res. 2016b: E-pub ahead of print. doi:10.1519/JSC.0000000000001477

Malone S, Roe M, Doran DA, Gabbett TJ, Collins KD. Aerobic Fitness and Playing Experience Protect Against Spikes in Workload: The Role of the Acute:Chronic Workload Ratio on Injury Risk in Elite Gaelic Football. Int J Sports Physiol Perform. 2016b; In Press

Malone S, Roe M, Doran DA, Gabbett TJ, Collins K. High chronic training loads and exposures to bouts of maximal velocity running reduce injury risk in elite Gaelic football. J Sci Med Sport 2016c; pii: S1440-2440(16)30148-30157. doi: 10.1016/j.jsams.2016.08.005

Mourot L, Bouhaddi M, Tordi N, Rouillon JD, Regnard J. Short- and long-term effects of a single bout of exercise on heart rate variability: comparison between constant and interval training exercises. Eur J Appl Physiol 2004; 92(4-5):508-517.

Pitchford N, Robertson S, Sargent C, Cordy F, Bishop DJ, Bartlett JD. A change in training environment alters sleep quality but not quantity in elite Australian Rules Football players. Int J Sports Physiol Perform. 2016; In Press.

Ritchie D, Hopkins WG, Buchheit M, Cordy J, Bartlett JD. Quantification of training and competition load across a season in an elite Australian football club. Int J Sports Physiol Perform 2016; 11(4): 474-479.

Rogalski B, Dawson B, Heasman J, Gabbett TJ. Training and game loads and injury risk in elite Australian footballers. J Sci Med Sport. 2013;16(6):499-503. 
Thornton HR, Duthie GM, Pitchford NW, Delaney JA, Benton DT, Dascombe BJ. Effects of a two-week high intensity training camp on sleep activity of professional rugby league athletes. Int J Sports Physiol Perform. 2016; In Press

Thorpe RT, Strudwick AJ, Buchheit M, Atkinson G, Drust B, Gregson W. Monitoring fatigue during the in-season competitive phase in elite soccer players. Int J Sports Physiol Perform. 2015; 10, 958-964. doi:10.1123/ijspp.2015-0004

Tran J, Rice AJ, Main LC, Gastin PB. Convergent validity of a novel method for quantifying rowing training loads $J$ Sports $S c i$ 2015; 3: 268-276. doi:

600 $10.1080 / 02640414.2014 .942686$

601

602

603

604

605

606

607

608

609

610

611

612

613

614

615

616

617

618

619

620

621

622

623

624

625

626 


\section{FIGURE CAPTIONS}

Figure 1 - Daily changes in (A) total distance (m) - double bars indicate completion of two sessions on the given day, (B) training load (sRPE; AU) - double bars indicate completion of two sessions on the given day, (C) sub-maximal exercise heart rate (HRex) and Heart rate recovery (HRR), (D) natural logarithm of standard deviation of instantaneous beat-to-beat R$\mathrm{R}$ interval variability, measured from Poincaré plots prior to the completion of training (LnSD1). All data presented as mean \pm SD.

Figure 2 - Daily changes in (A) wellness (AU), (B) sleep quality (AU) (C) countermovement jump (cm) (D) maximal velocity $\left(\mathrm{km} \cdot \mathrm{h}^{-1}\right)(\mathrm{E})$ fatigue $(\mathrm{F})$ stress. All data presented as mean $\pm \mathrm{SD}$.

Figure 3 - Running performance changes during and after the camp as measured by total distance during the Yo-Yo Intermittent Recovery Level 1 (Yo-YoIR1) and total (TD) and high-speed (HSD) and sprint (SPD) distance during standardized small sided games (SSG).

Figure 4 - Correlation coefficients ( $90 \%$ confidence intervals, CI) between daily (i.e. sessionto-session) changes in training load ( $\Delta \mathrm{TL})$ and daily (i.e. session-to-session) changes in submaximal exercise heart rate $(\Delta \mathrm{HRex})$, heart rate recovery $(\Delta \mathrm{HRR})$, natural logarithm of standard deviation of instantaneous beat-to-beat $\mathrm{R}-\mathrm{R}$ interval variability, measured from Poincaré plots prior to training $(\Delta \operatorname{LnSD} 1)$, perceived fatigue ( $\Delta$ Fatigue), sleep quality $(\Delta$ Sleep), muscle soreness $(\Delta$ Soreness $)$, stress $(\Delta$ Stress $)$, energy ( $\Delta$ energy) during the training camp.

\section{TABLE CAPTIONS}

Table 1 - The correlation coefficients $(90 \% \mathrm{CI})$ between daily individual changes in submaximal exercise heart rate $\left(\Delta \mathrm{HR}_{\mathrm{ex}}\right)$, heart rate recovery $(\Delta \mathrm{HRR})$, natural logarithm of standard deviation of instantaneous beat-to-beat $\mathrm{R}-\mathrm{R}$ interval variability, measured from Poincaré plots prior to training ( $\triangle \mathrm{LnSD} 1)$, overall wellness ( $\Delta$ Wellness) and running performance changes during and after the camp as measured by total distance during the Yo-Yo Intermittent Recovery Level 1 (Yo-YoIR1) performed outdoor and total distance, high-speed distance (HSD) and sprint (SPD) distance during standardized SSG performed outdoor. 

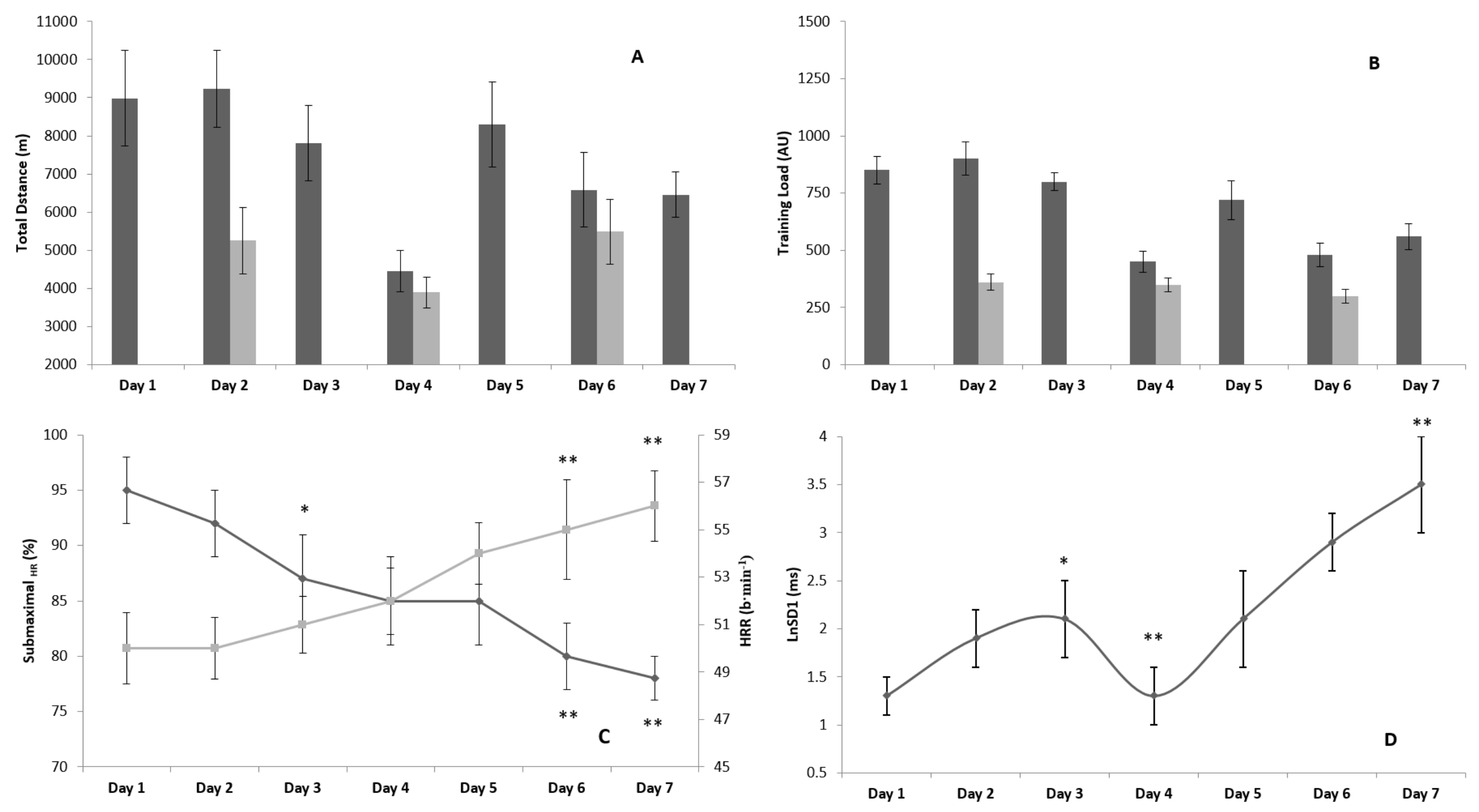

*Significant difference vs. day one with $P<0.05$. **Significant difference vs. day one with $P<0.01$ 
675

$676 \quad$ Figure 2 

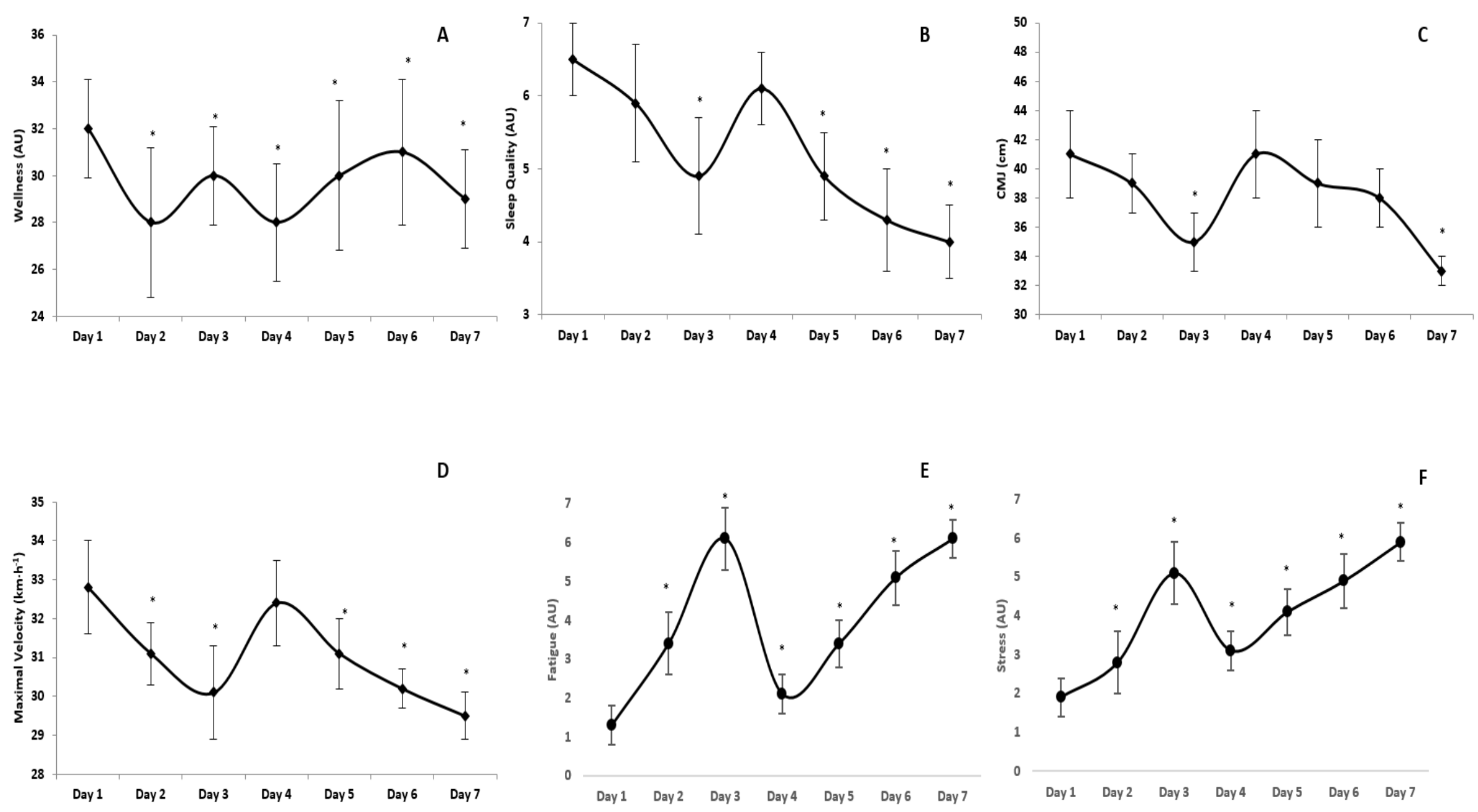
Figure 3

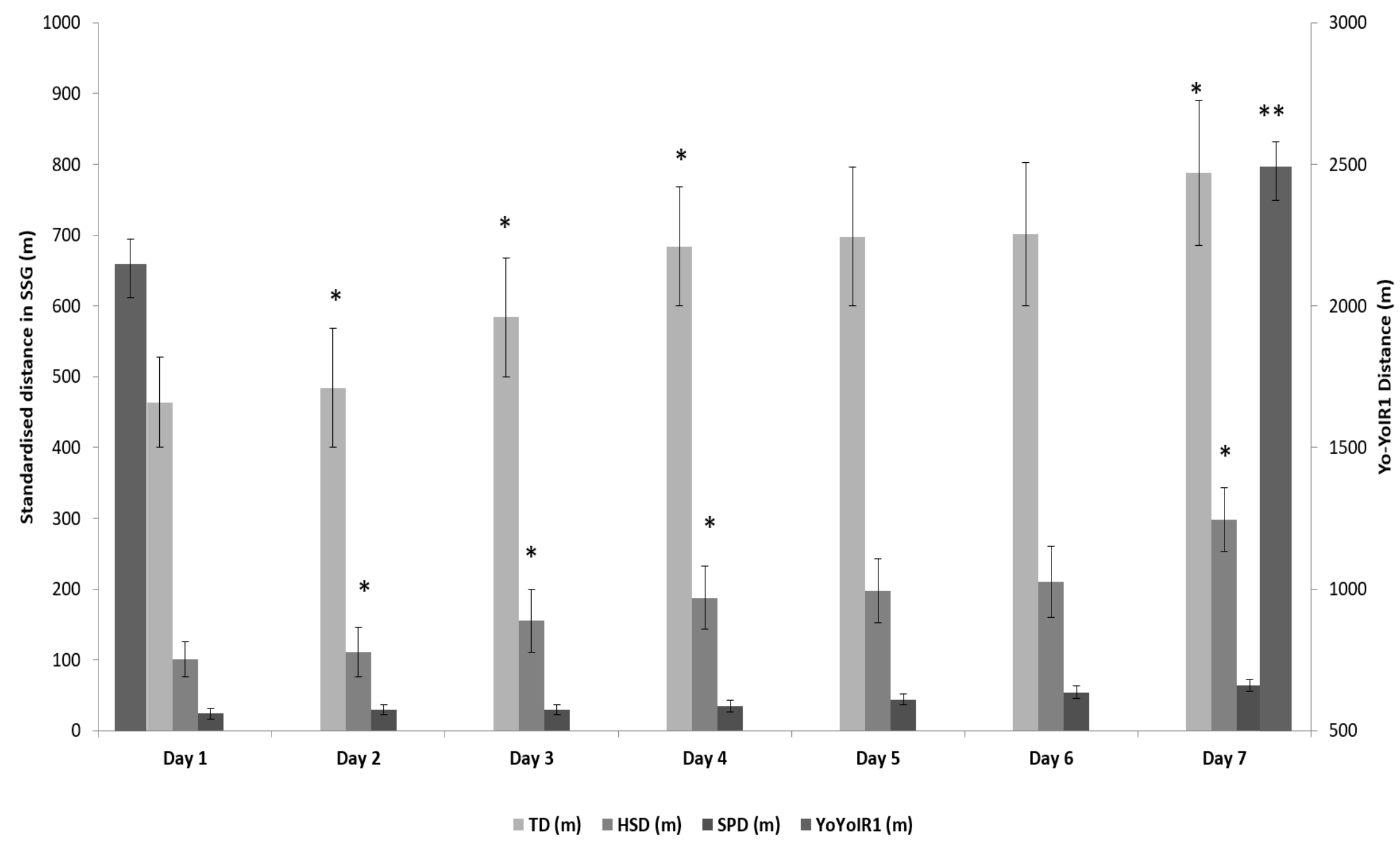




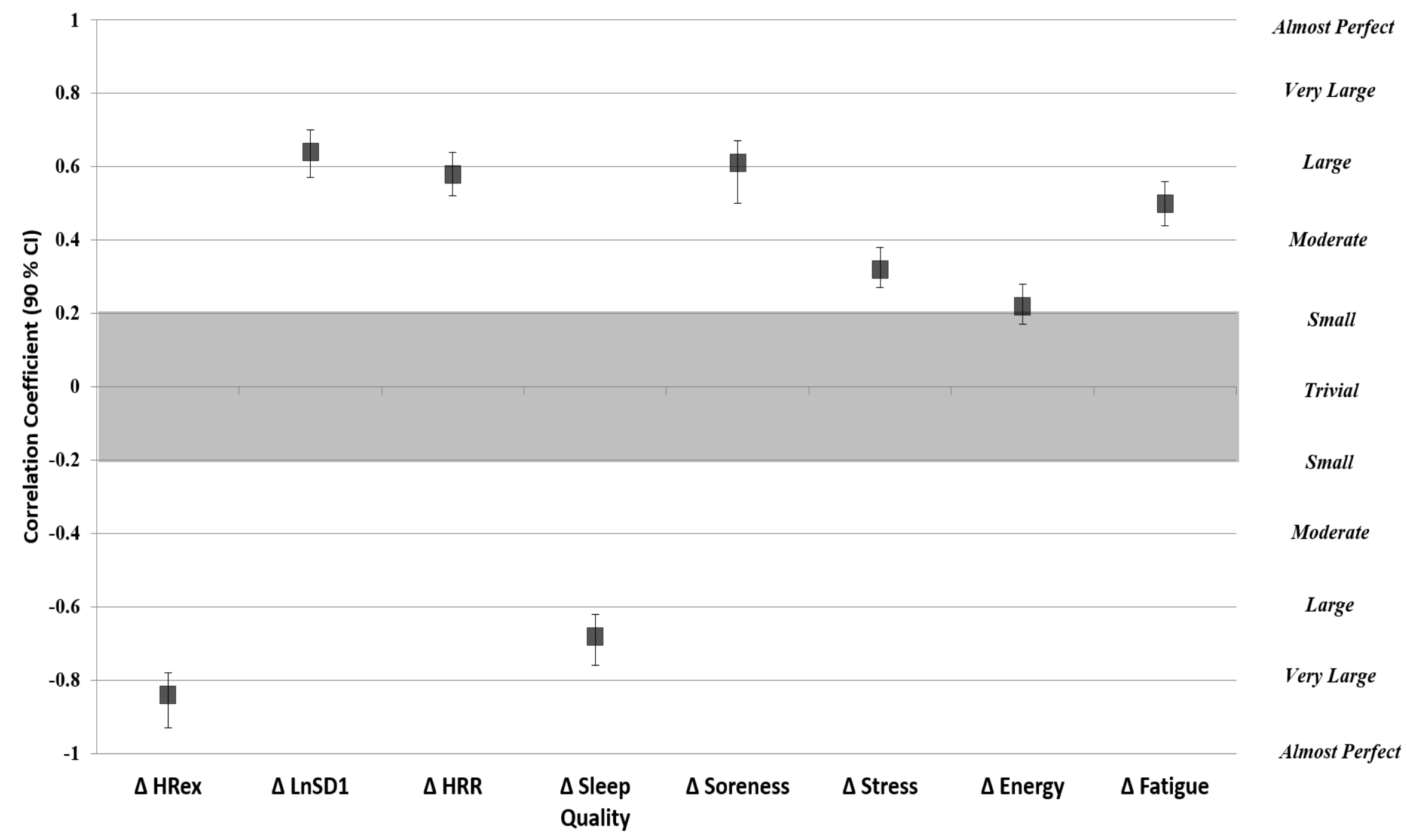


Table 1

686

\begin{tabular}{ccccc}
\hline & $\Delta$ LnSD1 & $\Delta$ HRR & $\Delta$ HR $_{\text {ex }}$ & $\Delta$ Wellness \\
\hline Yo-YoIR1 (m) & $0.48(0.33 \text { to } 0.71)^{* *}$ & $-0.55(-0.33 \text { to }-0.71)^{*}$ & $0.64(0.44 \text { to } 0.78)^{* *}$ & $0.71(0.55 \text { to } 0.87)^{* *}$ \\
TD (m) & $0.71(0.55 \text { to } 0.87)^{* *}$ & $-0.10(-0.25$ to -0.05$)$ & $0.20(0.11$ to 0.38$)$ & $0.68(0.45 \text { to } 0.66)^{* *}$ \\
HSD (m) & $0.19(0.09$ to 0.29$)$ & $-0.58(-0.32 \text { to }-0.78)^{*}$ & $0.44(0.11 \text { to } 0.65)^{*}$ & $0.17(0.05$ to 0.22$)$ \\
SPD (m) & $0.22(0.12$ to 0.32$)$ & $-0.52(-0.33 \text { to }-0.76)^{*}$ & $0.58(0.33$ to 0.66$)$ & $0.68(0.53 \text { to } 0.77)^{* *}$ \\
\hline
\end{tabular}

687 